\title{
Microbial diversity analysis of vineyard son the eastern foothills of the Helan Mountain region using high-throughput sequencing
}

\author{
Bei XUE , Jiajun $\mathrm{YU}^{2}$, Jiachen $\mathrm{ZHANG}^{1}$ (D), Feike $\mathrm{HAO}^{2}$, Xiaomeng $\mathrm{ZHANG}^{2}$, Jianhui $\mathrm{DONG}^{2}$, Jinyuan $\mathrm{SUN}^{3 *}$, \\ Jie XUE ${ }^{2,4 *}(1)$
}

\begin{abstract}
In this study, Illumina MiSeq was used to investigate the microbial diversity ofwine grapes and soil from three vineyards indifferent localities on the eastern foothills of Helan Mountain in Ningxia, China. The results of 26 samples showed thatthere were 4 fungal phyla with 1107 operational taxonomic units (OTUs) and 11 bacterial phyla with 4630 OTUs. At the genus level, the dominant fungi in the grapes included Aspergillus, Alternaria, Exobasidum, Aureobasidium, and Cladosporium, while the dominant fungi in the soil included Gibberella, Gliomastix, Coprinellus, Fusarium, and Chaetomium, Chryseobacterium, Mesorhizobium, Pseudomonas, Microbacterium and Acinetobacter were the dominant bacteria in the grapes, while Arthrobacter, Pontibacter, Massilia, Skermanella, and Candiatus-Nitrosocosmicus were the dominant bacteria in the soil. Principal component analysis showed that the microbial diversity differed in the three vineyards from different geographical locations. This study provides a theoretical basis for the utilization and development of the characteristic microorganisms in vineyards.
\end{abstract}

Keywords: the eastern foothills of Helan Mountain wine region; high-throughput sequencing; microbial diversity; wine grapes; soil.

Practical Application: High throughput analysis of microbial diversity was used.

\section{Introduction}

The regions of Heilongjiang, Shandong, the Yellow River delta, Hebei, Shanxi, Gansu, Ningxia, the Yellow River old riverway, Xinjiang, and the Yunnan plateau are 10 well-known grape-producing regions with regional characteristics, with the grape cultivation area and output accounting for more than $80 \%$ of the country. The wine industry in Yinchuan in the Helan Mountain region of Ningxia has developed rapidly in recent years and is known as the "star region" and "new region" in the international wine industry. This recognition by the international wine industry continues to draw attention to the region.

The flavor properties of wine are not only influenced by the geographical location, soil type, and climate, but are also closely related to the microorganisms involved in the fermentation of wine. Correlational studies have shown that White table wines are diuretic, red wines are more nutritious and tonic (Sarkisian, 2019). The unique flavor of wine is mainly produced by microbial metabolism during the wine brewing process. These microorganisms mainly come from the grape itself, the vineyard, or the environment of the production workshop. Determining the microbial resources on the grape surface as well as the ecology of microorganisms in the vineyard are of great importance. Current analyses of the microbial diversity of the grape and wine production environment have typically used traditional cultivation methods combined with polymerase chain reaction (PCR), restriction fragment length polymorphism (RFLP), and random amplified polymorphism analysis technique (RAPD) technologies. However, these methods are laborious, time-consuming, and exhibit poor reliability, which has restricted their large-scale application in microbial diversity analysis. With the development of science and technology, high-throughput sequencing technology has been increasingly applied to the study of microbial diversity. High-throughput sequencing technology has the characteristics of high DNA flux, high data accuracy and data large volumes, short time requirements, and low relative costs (Cocolin et al., 2000; Prakitchaiwattana et al., 2004; Renouf et al., 2007). Kioroglou et al. found that oak barrel aging had no significant effect on microbial diversity during the wine aging process, but did alter the population structure and composition of fungi and bacteria (Ma et al., 2018). Using highthroughput sequencing technology, Wang et al. (2020b) found that inoculation with starter cultures altered the interactions and metabolism of primitive fungal communities during pomegranate wine fermentation. Therefore, not only the positive characteristics of the starter culture, but also the interaction between the starter culture and the original flora should be considered in starter culture inoculation (Kioroglou et al., 2020). Wang et al. (2020b) analyzed the traditional fungal resources 
in different regions of Guizhou, China, by high-throughput sequencing technology and found that two strains of yeast, namely FBKL2.8022 and FBKL2.8023, were found to have low yields of higher alcohols and could produce $11.70 \% \mathrm{vol}$ and $7.10 \%$ vol ethanol separately. However, there are few reports on the application of high-throughput sequencing technology in the analysis of wine microbial diversity in the wine-producing areas of the eastern foothills of Helan Mountain.

In this study, high-throughput sequencing technology was used to analyze the microbial composition of wine grapes and soil in LiLan (LL), ZhenBeiPu (ZBP), and GanChengZi (GCZ) three vineyards on the eastern foothills of Helan Mountain. The microbial community structure of the area was analyzed in order to reveal the microbial diversity and the differences in microbial community structure, provide theoretical guidance for the study of the laws of microbial interactions in wine fermentation, and to provide a theoretical basis for the screening of high-quality microorganisms and the establishment of a microbial resource library for wine making on the eastern foothills of Helan Mountain.

\section{Materials and methods}

\subsection{Material}

The samples were collected from the grape berries and soil of the upper ZBP, middle LLand nether GCZ vineyards on the eastern foothills of Helan Mountain, Ningxia, China. The sampling time was September 2019. The specific samples are shown in Table 1.

\subsection{Test methods}

\section{Sample collection method}

Soil sampling method: Plant residues were removed from the soil surface, the soil was dug vertically with a soil shovel, and about $0.5 \mathrm{~kg}$ of soil was obtained for each sampling point at a depth of $20 \mathrm{~cm}$ (Wang et al., 2020a).

Grape sampling method: The upper, middle, and lower parts of a vine were sampled, and the bunches of grapes were removed with scissors.

\section{Sample pretreatment}

Soil: The bulk soil was powdered, and $0.4 \mathrm{~g}$ of ground soil sample and $0.5 \mathrm{~g}$ of grinding beads were weighed in a sterilized $2 \mathrm{~mL}$ centrifuge tube.

Wine grape berries: First, the peeled grape skins were ground in liquid nitrogen, and $4 \mathrm{~g}$ of the ground skins was added to

Table 1. Sample information sheet.

\begin{tabular}{clc}
\hline Sample & \multicolumn{1}{c}{ Number } & Region \\
\hline \multirow{4}{*}{ soil } & R1(T1、T2、T3) & LL \\
& R2(T4、T5、T6) & ZBP \\
& R3(T7、T8、T9) & GCZ \\
& A1(AP1、AP2、AP3、AP4、AP5、AP6) & LL \\
grape & A2(BP1、BP2、BP3、BP4、BP5) & ZBP \\
& A3(CP1、CP2、CP3、CP4、CP5、CP6) & GCZ \\
\hline
\end{tabular}

a clean $50 \mathrm{~mL}$ centrifuge tube, to which $6 \mathrm{~mL}$ cell lysis buffer TENP $(50 \mathrm{mM}$ Tris, $20 \mathrm{mM}$ EDTA, $100 \mathrm{mM} \mathrm{NaCl}, 0.01 \mathrm{~g} / \mathrm{mL}$ PVP pH=10) solution was added (Renouf et al., 2007) and the solution centrifuged at $5000 \mathrm{~g}$ for $7 \mathrm{~min}$. The supernatant was then transferred to a new centrifuge tube, and the previous step was repeated. The supernatant was carefully transferred to the centrifuge tube and centrifuged at $9000 \mathrm{~g}$ for $10 \mathrm{~min}$. The supernatant was discarded, and the obtained precipitate was used as the sample and was stored at $-20^{\circ} \mathrm{C}$ for later use.

\section{High-throughput sequencing analysis of the samples}

Extraction of sample DNA

A Mag-bind Soil DNA Kit Protocol Kit (OMEGA) was used to extract the original microbial genomic DNA from the soil. A Fast DNA SPIN Kit for Soil Kit (MP) was used to extract the original genomic DNA of the microorganisms on the grape surface.

Sample PCR (Boulton et al., 1999; Liu et al., 2021)

The general primers for ITS1 amplification of the fungi included ITS1F (5'-CTTGGtCatttAGAgGagtaa-3') and ITS1R (5'-GCTGCGTTCTTCATcGATGC-3'). The PCR $(50 \mu \mathrm{L})$ amplification system included $4 \mu \mathrm{L}$ dNTP Mixture (Takara, China), $5 \mu \mathrm{L} 10 \times$ PCR Buffer (Mgel Plus) (Takara, China), $1 \mu \mathrm{L}$ of forward and reverse primers (Sangon, China), $5 \mu \mathrm{L}$ sample DNA, and $0.25 \mu \mathrm{L}$ ExTaq enzyme, with $\mathrm{dd}_{2} \mathrm{O}$ added to a volume of $50 \mu \mathrm{L}$. The solution was well mixed. The PCR amplification procedure included 35 cycles of pre-denaturation at $98^{\circ} \mathrm{C}$ for 3 min, denaturation at $98^{\circ} \mathrm{C}$ for $45 \mathrm{~s}$, annealing at $53^{\circ} \mathrm{C}$ for $30 \mathrm{~s}$, and elongation at $72{ }^{\circ} \mathrm{C}$ for $45 \mathrm{~s}$, followed by an elongation step of $72{ }^{\circ} \mathrm{C}$ for $8 \mathrm{~min}$. The mixture was stored at $-20^{\circ} \mathrm{C}$ until later use.

The general primers for bacterial 16SrDNAV4 amplification included 16S515F (5'-GTGCCAGCMgCCGCGGTAA-3) and 16S806R (5'-GGactachvGGGTWTCTAAT-3'). The PCR $(50 \mu \mathrm{L})$ amplification system included $4 \mu \mathrm{L}$ (dNTP Mixture Takara, China), $5 \mu \mathrm{L} 10 \times$ PCR Buffer $(\mathrm{Mg})^{2+}$ Plus (Takara, China), $1 \mu \mathrm{L}$ each of forward and reverse primers (Sangon, China), $5 \mu \mathrm{L}$ template DNA, and $0.25 \mu \mathrm{L}$ ExTaq, with ddH2O added to $50 \mu \mathrm{L}$ and mixed well. The PCR amplification procedure included 35 cycles of pre-denaturation at $95^{\circ} \mathrm{C}$ for $3 \mathrm{~min}$, denaturation at $95^{\circ} \mathrm{C}$ for $45 \mathrm{~s}$, annealing at $50^{\circ} \mathrm{C}$ for $30 \mathrm{~s}$, and elongation at $72{ }^{\circ} \mathrm{C}$ for $1 \mathrm{~min}$, followed by an elongation step at $72{ }^{\circ} \mathrm{C}$ for $5 \mathrm{~min}$. The mixture was stored at $-20^{\circ} \mathrm{C}$ for later use.

Analysis of PCR products by agarose gel electrophoresis

Using a DL2000 Marker and 1\% agar-agar gel, electrophoresis of PCR products was performed at $110 \mathrm{~V}$ constant pressure for $35 \mathrm{~min}$, and a gel imager was used to observe the electrophoresis results.

High-throughput sequencing (Zhan et al., 2011; Martins et al., 2012; Verginer et al., 2010; Wawrik et al., 2005; Feng et al., 2003)

The PCR products of the fungi and bacteria were extracted with an AxyPrep DNA gel recovery kit. A Life Qubit 3.0 was 
used to quantify the concentration of the purified PCR products, and the regional libraries of fungal ITS1 and bacterial 16SrDNA $\mathrm{V} 4$ were constructed. The sample (library) was gradually diluted to $4 \mathrm{~nm}$, and sodium hydroxide was added at a 1:1 ratio and denatured at room temperature for $5 \mathrm{~min}$. HT1 buffer was added for pre-cooling, and 8 PPM PCR amplification products were selected for high-throughput on-machine sequencing.

Construction of the DNA library: A transposon Tn5 was used for high-throughput sequencing. By recognizing the target sequence of the recipient's DNA, the recipient's DNA was removed, and the donor DNA was inserted into the donor DNA to form a P5 connector at one end and a P7 connector at the other end, and a complete connector DNA library containing the p5 terminal and P7 terminal was formed through PCR technology.

Flow cell adsorption: Complementary sequences of the P5 and P7 joints were distributed in the flow cell. Different indexes were added to each sample under test to generate reads with the DNA of different samples. Thus, the DNA sequencing data of the different samples could be obtained separately.

Bridging PCR amplification: The DNA sequence to be measured was hybridized and complemented with the sequence on the flow cell through the connector sequence. The DNA sequence to be measured was used as a template for complementary chain extension, and then the template chain was cut off and washed down for removal. The complementary chain was then crossbred with the joint sequence on the flow cell to complement the synthesis.

Sequencing by synthesis (SBS): The newly synthesized end markers were captured to determine the DNA sequence.

\section{Sequencing data statistics}

Pairing-end sequencing V1.2.7 (CCB, 2020) was used to discard low-quality sequences in the original data (the average mass of 50 consecutive bases was guaranteed to be greater than Q30). Flash software was used to filter the docking sequences (continuous identical base number less than six, fuzzy base number less than one), design the undocking sequences, and finally obtain them for use in operational taxonomic unit (OTU) identification Sequence analysis.

\section{Generation of the OTU list}

Qiime software V1.9.1 (Qiime, 2020a) was used to classify the sequences with a similarity of more than $97 \%$ into OTUs. All sequences were clustered using the Ucl method based on the RDP (Ribosomal Database Project) Database, and a Bayesian algorithm was used to annotate the OTU representing the sequence in each classification, and the taxonomic information of each OTU was obtained.

\section{Analysis of microbial community structure and diversity}

The OTU data were sorted (Qiime, 2020b), and a species abundance histogram was created for fungi and bacteria in each sample to determine the dominant bacteria genera. The similarity between samples was analyzed by principal component analysis and clustering analysis. The same genera of bacteria in the LL, ZBP, and GCZ samples in three vineyards were observed in the Venn diagram, allowing for a comprehensive analysis of the diversity of the microbial community in each sample.

\section{Results and analysis}

\subsection{Microbial community diversity}

\section{Bacterial community diversity of the samples}

The high-throughput sequencing results (Drive5, 2020) of the 26 samples in this study showed that a total of 4,630 bacterial microorganisms were found at the phylum level. Due to the wide variety of microorganisms in the samples, bacterial species with an abundance greater than $1 \%$ were drawn, as indicated in Figure 1. The most abundant bacterial phyla in the grape skins were Proteobacteria, Bacteroidetes, Actinobacteria, Firmicutes, Deinococcus-Thermus, and Fusobacteria. Proteobacteria was the most abundant genus, and Bacteroidetes was the second most abundant genus in the grape samples. The quantity of Bacteroidetes was relatively stable in LL and GCZ, but lower in $\mathrm{ZBP}$. One possible reason is that the average annual temperature in $\mathrm{ZBP}$ area is low, and the optimum growth temperature for Bacteroidetes is $37^{\circ} \mathrm{C}$. Deinococcus-Thermus was present in LL and GCZ, but not in ZBP. Actinobacteria, Proteobacteria, Unidentified Bacteria, Bacteroidota, and Crenarchaeota were the dominant phyla in the soil. In addition, Firmicutes and Cyanobacteria were also found in the soil of LL, but were only present in small amounts in ZBP and GCZ.

Microbial alpha diversity indexes include the Shannon (Mothur, 2020a) Simpson (Mothur, 2020b), chao1 (Mothur, 2020c), ACE (Mothur, 2020d), and Coverage (Mothur, 2020e) diversity indexes. The greater Shannon value, the higher the community diversity. Coverage refers to the coverage of each sample library, and a higher value indicates a higher probability that the sequence in the sample will be measured, whereas a lower the probability indicates that it will not be measured. As indicated in Table 2, the coverage of all samples was $99.9 \%$, but there were significant differences in the Shannon diversity indexes of the different vineyard samples. As shown in Table 2, the Shannon index of T3 in LL the vineyard soil samples was the highest at 9.409, indicating that its community diversity was the most abundant. The Shannon index of AP4 was 3.458 in the grape samples, indicating that its community diversity was the most abundant. In the soil samples of ZBP, the Shannon index of T4 was the highest at 8.983, indicating abundant community diversity. The Shannon index of BP4 in the grape samples was as high as 3.177, indicating that its community diversity was the highest. In the GCZ soil samples, the Shannon index of T7 was the highest at 8.861 , indicating abundant community diversity. The highest Shannon index of CP4 in the grape samples was 4.015 , indicating the highest diversity in its community.

\section{Diversity of fungal communities in the samples}

The high-throughput sequencing results of 26 samples in this study showed that 1107 fungal microorganisms were found at the phylum level, as shown in Figure 2. The strains Ascomycota, Basidiomycota, Mortierellomycota, Chytridiomycota, 


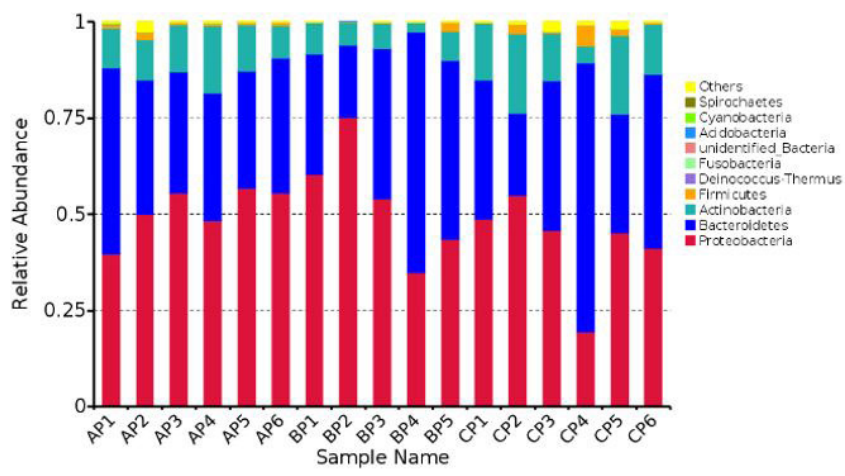

(a)grape

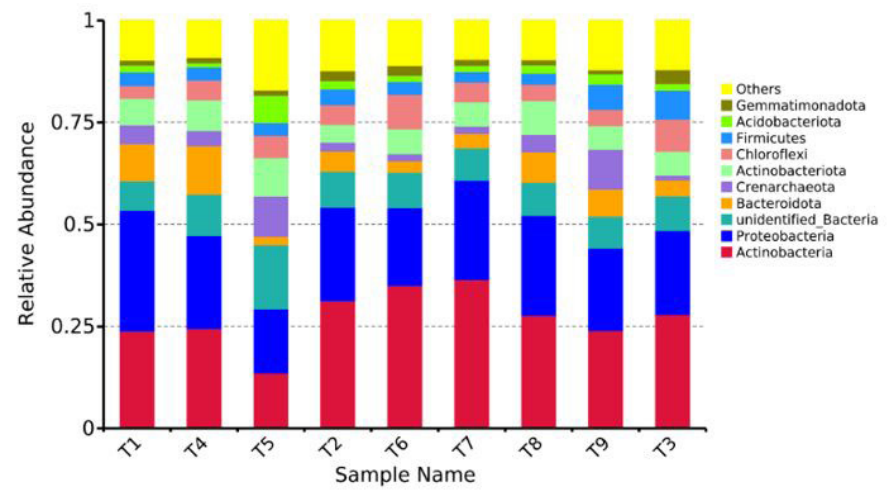

(b)soil

Figure 1. Distribution of 10 of the most abundant bacterial genera in the soil and grape samples collected from the three vineyards. "Others" in the last column refers to the sum of detected minority fungal genera. Each number represents the average of three replicates.

Table 2. Sample statistics of the alpha indices.

\begin{tabular}{|c|c|c|c|c|c|c|c|c|c|c|}
\hline \multirow{2}{*}{ Sample Name } & \multicolumn{2}{|c|}{ shannon } & \multicolumn{2}{|c|}{ simpson } & \multicolumn{2}{|c|}{ chaol } & \multicolumn{2}{|c|}{$\mathrm{ACE}$} & \multicolumn{2}{|c|}{ goods_coverage } \\
\hline & bacterial & fungal & bacterial & fungal & bacterial & fungal & bacterial & fungal & bacterial & fungal \\
\hline $\mathrm{T} 1$ & 8.705 & 3.972 & 0.99 & 0.774 & 2724.686 & 335.319 & 2747.909 & 341.17 & 0.999 & 0.999 \\
\hline $\mathrm{T} 2$ & 8.572 & 4.92 & 0.992 & 0.912 & 2291.031 & 457.568 & 2311.643 & 453.557 & 0.999 & 0.999 \\
\hline T3 & 9.409 & 4.155 & 0.995 & 0.797 & 3012.552 & 401.475 & 3033.906 & 410.169 & 0.999 & 0.999 \\
\hline $\mathrm{T} 4$ & 8.983 & 4.546 & 0.991 & 0.89 & 3774.536 & 387.775 & 3401.836 & 387.296 & 0.999 & 0.999 \\
\hline T5 & 8.848 & 4.001 & 0.993 & 0.848 & 2741.5 & 366.926 & 2796.466 & 377.397 & 0.999 & 0.999 \\
\hline T6 & 8.268 & 5.606 & 0.986 & 0.949 & 2233.668 & 537.514 & 2265.835 & 544.626 & 0.999 & 0.999 \\
\hline $\mathrm{T} 7$ & 8.861 & 4.915 & 0.992 & 0.904 & 2632.799 & 473.721 & 2651.775 & 481.116 & 0.999 & 0.999 \\
\hline $\mathrm{T} 8$ & 8.726 & 4.762 & 0.99 & 0.902 & 2816.281 & 493.152 & 2859.34 & 504.998 & 0.999 & 0.999 \\
\hline T9 & 8.831 & 4.877 & 0.992 & 0.91 & 2472.682 & 478.922 & 2509.588 & 484.266 & 0.999 & 0.999 \\
\hline AP1 & 3.011 & 3.536 & 0.758 & 0.828 & 203 & 333.458 & 201.706 & 340.484 & 0.999 & 0.999 \\
\hline AP2 & 3.4 & 2.975 & 0.801 & 0.743 & 277.69 & 282.448 & 278.834 & 293.359 & 0.999 & 0.999 \\
\hline AP3 & 2.903 & 3.124 & 0.77 & 0.764 & 156.625 & 308.062 & 161.054 & 320.643 & 0.999 & 0.999 \\
\hline AP4 & 3.458 & 3.282 & 0.829 & 0.805 & 203 & 309.85 & 202.51 & 320.242 & 0.999 & 0.999 \\
\hline AP5 & 2.752 & 3.736 & 0.742 & 0.843 & 179.8 & 353.672 & 185.567 & 363.284 & 0.999 & 0.999 \\
\hline AP6 & 2.871 & 2.986 & 0.825 & 0.635 & 72.438 & 519.794 & 76.212 & 518.843 & 0.999 & 0.999 \\
\hline BP1 & 2.547 & 2.101 & 0.754 & 0.568 & 73.5 & 224.207 & 78.855 & 224.396 & 0.999 & 0.999 \\
\hline BP2 & 2.844 & 0.579 & 0.812 & 0.136 & 100.714 & 104.636 & 104.577 & 114.978 & 0.999 & 0.999 \\
\hline BP3 & 2.292 & 2.035 & 0.678 & 0.601 & 97 & 196.812 & 99.533 & 204.15 & 0.999 & 0.999 \\
\hline BP4 & 3.177 & 1.493 & 0.793 & 0.452 & 107 & 141.25 & 106.684 & 144.42 & 0.999 & 0.999 \\
\hline BP5 & 2.899 & 1.792 & 0.786 & 0.423 & 184.036 & 295.651 & 189.124 & 297.984 & 0.999 & 0.999 \\
\hline CP1 & 3.939 & 1.99 & 0.878 & 0.533 & 250.237 & 172.292 & 253.807 & 178.38 & 0.999 & 0.999 \\
\hline CP2 & 2.888 & 3.522 & 0.779 & 0.869 & 227.7 & 229.897 & 228.658 & 232.712 & 0.999 & 0.999 \\
\hline CP3 & 2.525 & 2.586 & 0.63 & 0.679 & 102 & 217.391 & 99.265 & 213.535 & 0.999 & 0.999 \\
\hline $\mathrm{CP} 4$ & 4.015 & 1.578 & 0.884 & 0.398 & 266.788 & 138 & 278.123 & 115.025 & 0.999 & 0.999 \\
\hline CP5 & 3.076 & 3.248 & 0.784 & 0.837 & 190.65 & 236 & 188.709 & 243.719 & 0.999 & 0.999 \\
\hline CP6 & 2.755 & 3.43 & 0.735 & 0.821 & 163.062 & 315.526 & 165.845 & 310.656 & 0.999 & 0.999 \\
\hline
\end{tabular}

and Glomeromycota were all present at higher proportions in LL, ZBP, and GCZ. Among them, Rozellomycota was more abundant in the grape samples, and Zoopagomycota was more abundant in the soil samples.
In the soil samples of LL, the Shannon value of T2 was as high as 4.92 , indicating that the community diversity was the highest. The Shannon index of AP5 in the grape samples was as high as 3.736 , indicating that its community diversity was the 


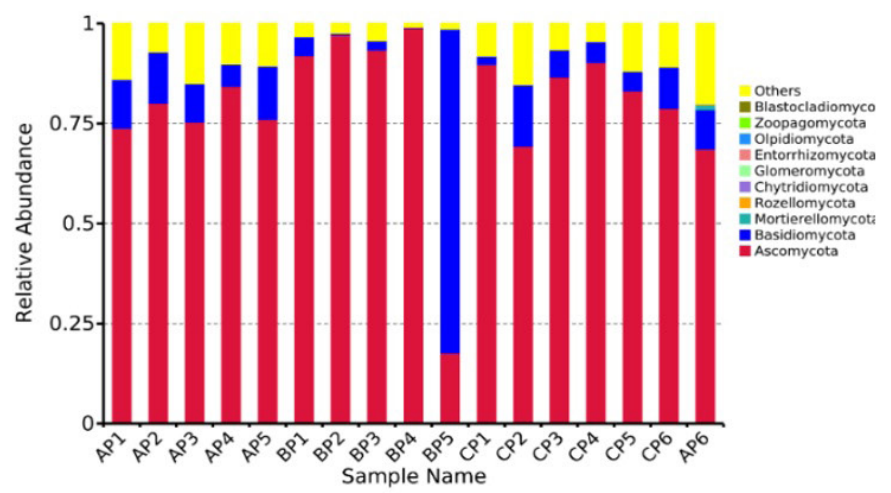

(a)grape

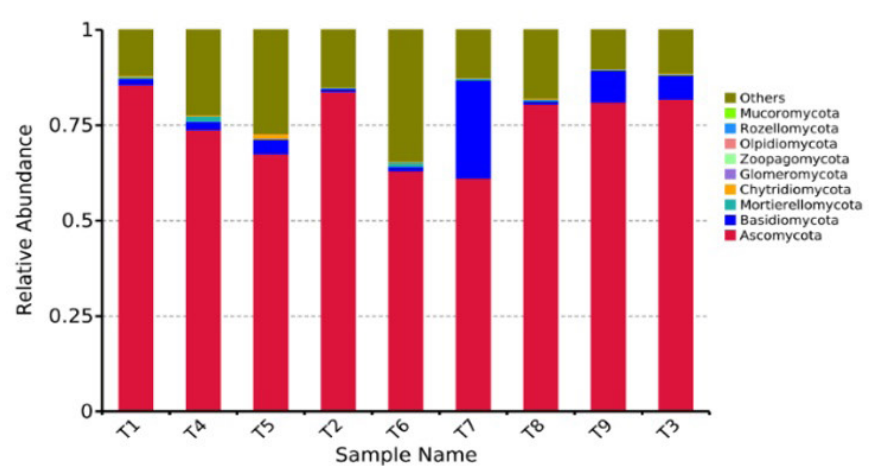

(b)soil

Figure 2. Distribution of 10 of the most abundant fungal genera in the soil and grape samples collected from the three vineyards. "Others" in the last column refers to the sum of detected minority fungal genera. Each number represents the average of three replicates.

most abundant. The Shannon value of T6 in the soil samples of ZBP was 5.606, indicating that the community diversity was the most abundant. The Shannon index of BP1 in the grape samples was 2.101 , indicating that its community diversity was the most abundant. In the GCZ vineyard soil samples, the Shannon of T7 was the highest at 4.915, indicating the most abundant microbial community diversity. In the grape samples, the Shannon index of CP2 was as high as 3.522, indicating that its microbial community was the most diverse.

\subsection{Analysis of microbial abundance}

Analysis of bacterial abundance in the samples

The relative abundance of bacteria in the sample cluster heatmap (Figure 3) showed that at the phylum level, the most abundant bacteria in the grape samples were Proteobacteria, Bacteroidetes, Actinobacteria, Firmicutes, and Deinococcus-Thermus.

At the generic level, Kocuria was abundant in the grape samples of AP2, Curtobacterium was relatively abundant in AP3, and Lactococcus, Stenotrophomonas, Plantibacter, Exiguobacterium, and Pseudoclavibacter were relatively abundant in BP5, of which Lactococcus is the key microorganism in malolactic fermentation (MLF). MLF can change the concentration of trace components such as aldehydes, esters, amino acids, other organic acids, and vitamins as well as the content of fragrant substances in wine (Kamaa et al., 2011). When high-throughput sequencing technology was used to analyze the bacterial community diversity of rice wine, Lactococcus was also found in the rice wine xiaqu. Bacillus, Herbaspirillum, and Gluconobacter were the most abundant CP2 microbes (Cappello et al., 2017). In CP4, the most abundant microbes were Micrococcus, Unidentified Corynebacteriaceae, Anaerococcus, and Staphylococcus. The most abundant microbe in CP5 was Kineococcus. The most abundant species in the soil samples at the phylum level were Actinobacteria, Proteobacteria, Unidentified Bacteria, Bacteroidota, and Crenarchaeota. At the genus level, Paracoccus, Lactobacillus, and Faecalibaculum were the most abundant in the soil samples in T3. Danielski et al. (2020) found that Lactobacillus improves the quality and safety of meat products (Zhao et al., 2020). Pontibacter was most abundant in T4; Rubrobacter was most abundant in T5; and Gemmatimonas had the highest abundance in T6. In T9, Dubosiella, Chthoniobacter, Candidatus-Nitrocosmicus, and Lachnospiraceae-Nk4A136-Group were all abundant.

The taxonomic information of the sample OTUs was illustrated in a Venn diagram using QIIME (Figure 4). According to the Venn diagram (Figure 4), the bacterial OTUs contained in the grapes of LL, ZBP, and GCZ were 397, 178, and 452, respectively. The LL, ZBP, and GCZ soil contained 3986, 3877, and 3638 bacterial OTU, respectively. According to the analysis of bacterial OTUs, the microbial surface abundance of the GCZ grape skins was the highest, while that of $\mathrm{ZBP}$ was the lowest. The reason may be that the $\mathrm{ZBP}$ is close to a lake, and the relatively abundant water can provide favorable conditions for the growth of microorganisms. The GCZ area is relatively short of water and has relatively low microbial abundance.

\section{Analysis of fungal abundance in the samples}

The relative abundance of the fungal clustering heatmap (Figure 5) showed that, at the phylum level, the most abundant fungi were Ascomycota, Basidiomycota, Mortierellomycota, Chytridiomycota, Glmeromyc, and Rozellomycota. At the genus level, Nigrospora, Saccharomycopsis, and Gibberella were the most abundant grape samples in A5. The AP6 samples showed high microbial diversity, with the highest abundance being Archaeorzomyces, Preussia, Hygrocybe, Mortierella, and Fusarium. Aspergillus was most abundant in BP2; and Phaeothecoidiella, Exobasidium, Unidentified cota-sp, and Hyaloscypha were the most abundant in BP5. Metschnikowia and Aureobasidium were most abundant in CP2, and Coniothyrium and Pichia were the most abundant in CP5. The most abundant strains in the soil samples at the phylum level included Ascomycota, Basidiomycota, Mortierellomycota, Chytridiomycota, Glmeromyc, and Zoopagomycota. Fusarium was the most abundant soil sample at $\mathrm{T} 1$ at the genus level. The T4 samples with the highest abundance included Talaromyces, Gliomastix, and Leptosphaeria. Rhizophlyctis, Stachybotrys, and Fusarium were the most abundant in T5. Unidentifiedchaetothyiales-SP, Unidentified-sordariomycetes-sp, Humicola, and Thysanorea were the most abundant in T6. 


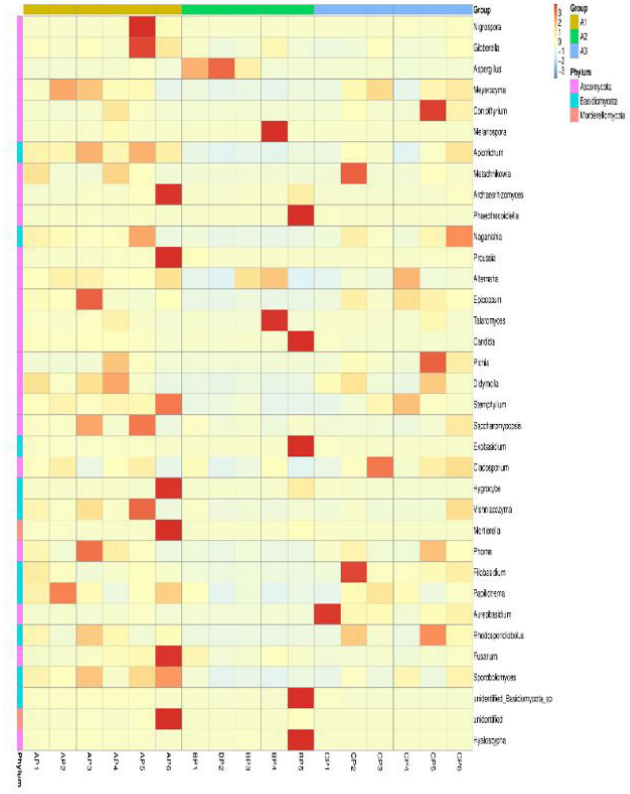

(a)grape

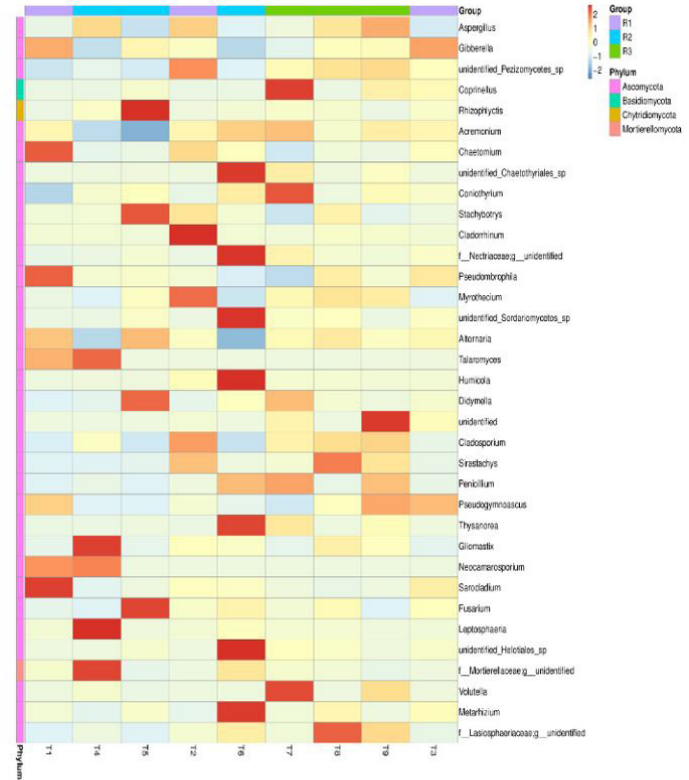

(b)soil

Figure 3. Relative abundance heatmap of bacteria in (a) grape samples and (b) soil samples.

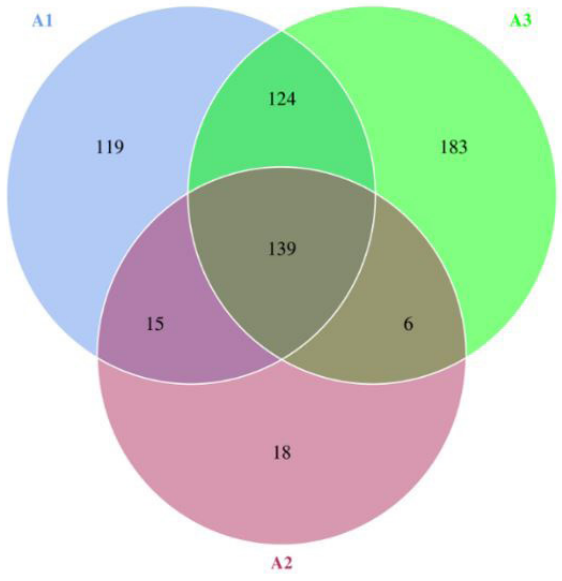

(a)grape

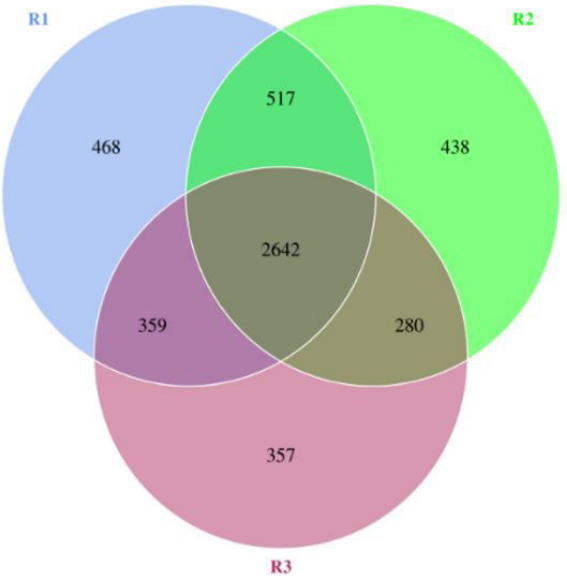

(b)soil

Figure 4. Venn diagram of the bacterial community in (a) grape samples and (b) soil samples.

Venn diagram analysis (Figure 6) showed that the OTU values of fungi in LL, ZBP, and GCZ were 980, 502, and 470, respectively. In the soil samples, the OTU values of the three wineries were 612,691, and 675, respectively. Among them, the OTU values of fungi in LL were higher than that in the soil, indicating that the fungal resources here were relatively abundant.

\subsection{Comparative analysis of the microbial communities in different samples}

The bacterial principal component analysis and cluster analysis of the samples are shown in Figure 7a. PC1 was 24.43\% and PC2 was $17.17 \%$, indicating that the microbial similarity on the surface of the grapes was relatively high, while the microbial similarity in the soil was relatively low. From the unweighted pair group method with arithmetic mean (UMPGA) cluster diagram (Figure 7b), the branches in grape samples had a value of 0.08 , indicating that the microbial difference between the grape samples was small. However, the branches in the soil samples had a value of 0.03 , indicating that the microbial difference between the soil samples was large.

The fungal principal component analysis and cluster analysis of the samples are shown in Figure 8a. PC1 was 18.38\% and PC2 was $16.6 \%$, indicating that the similarity of the fungal communities in grapes was relatively high, while the similarity in the soil was relatively low. The distance between the soil and grape samples on the plot is relatively far, suggesting low similarity. The LL and GCZ grape samples had the highest similarity. 


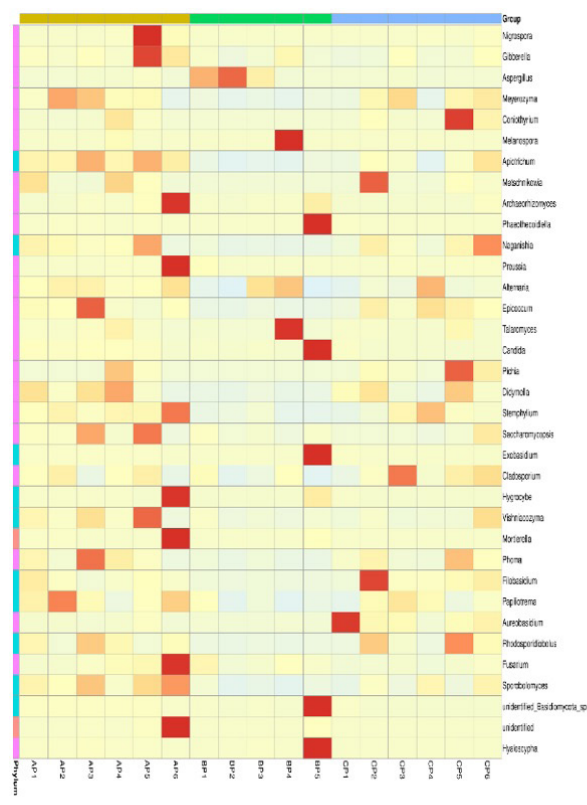

(a)grape
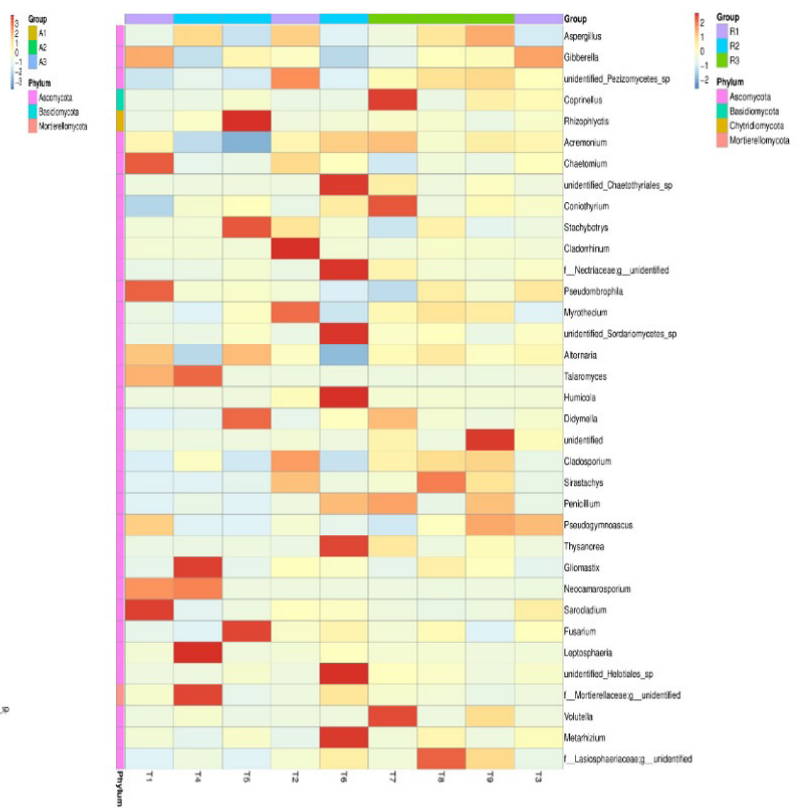

(b)soil

Figure 5. Relative abundance heatmap diagram of fungi in (a) grape samples and (b) soil samples.

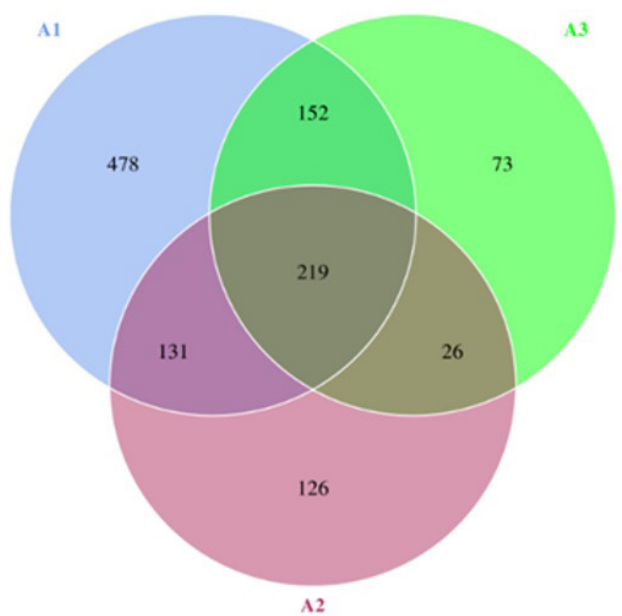

(a)grape

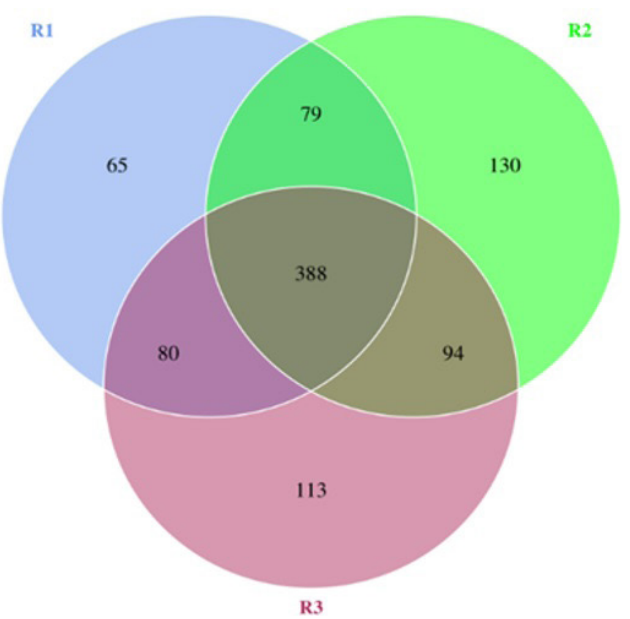

(b)soil

Figure 6. Venn diagram of the fungal community in (a) grape samples and (b) soil samples.

We further compared the microbial community differences among the samples from the different regions (Figure $8 \mathrm{~b}$ ). The fungi in the grape samples showed obvious branching at 0.15 , and the LL vineyard samples showed great differences, while the fungi in the grape samples in ZBP and GCZ showed no obvious differences. It can be seen from the soil clustering analysis diagram that obvious branches appeared at 0.076, indicating that the soil microorganisms in the three vineyards were quite different.

\section{Discussion}

With technological and scientific advancement, the study of wine microbes has extended from wine grape berries to the soil, leaves, grape bark, and tree roots of the vineyard ecosystem. Numerous species of fungi and bacteria have been found to be related to wine grapes (Danielski et al., 2020; Martins et al., 2012). In this study, second-generation Illumina MiSeq sequencing technology was used to analyze the diversity of microbial communities in the vineyards on the eastern foothills of Helan Mountain in Ningxia. The results showed that the microbial species in the soil were more abundant. Soil can provide a large number of nutrients needed for the growth of microorganisms, such as carbon sources (including amino acids, organic acids, and carbohydrates), nitrogen sources, and growth factors (Verginer et al., 2010). Compared with grape fruits and leaves, soil is rich and diverse in both fungi and bacteria, and it is a natural microbial reservoir. Feng et al. (2003) argue that this is mainly due to the fact that artificial tillage practices, such as fertilization, drug application, no-till, crop 


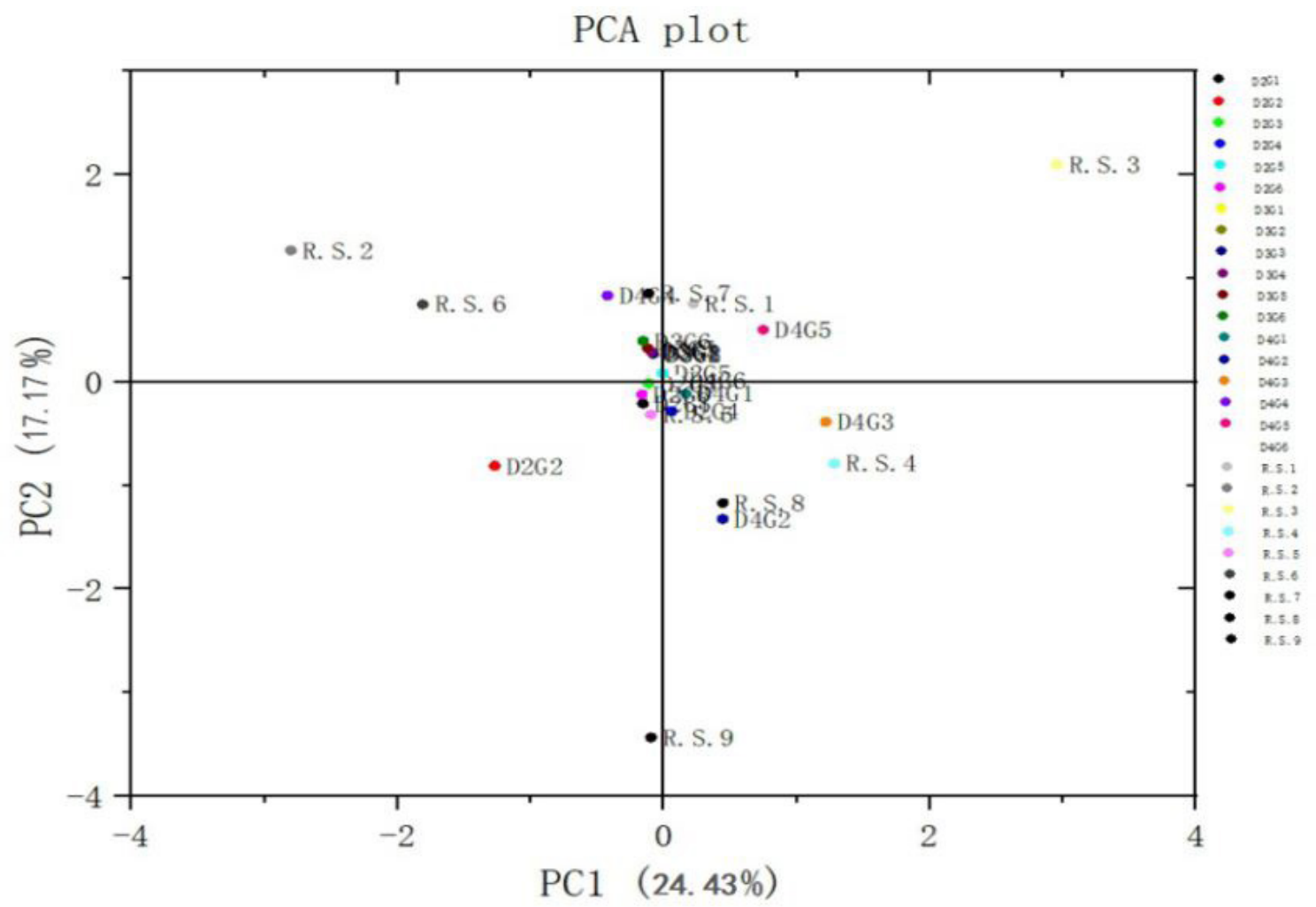

Figure 7a. Principal component analysis plot of the bacterial community in the different samples.

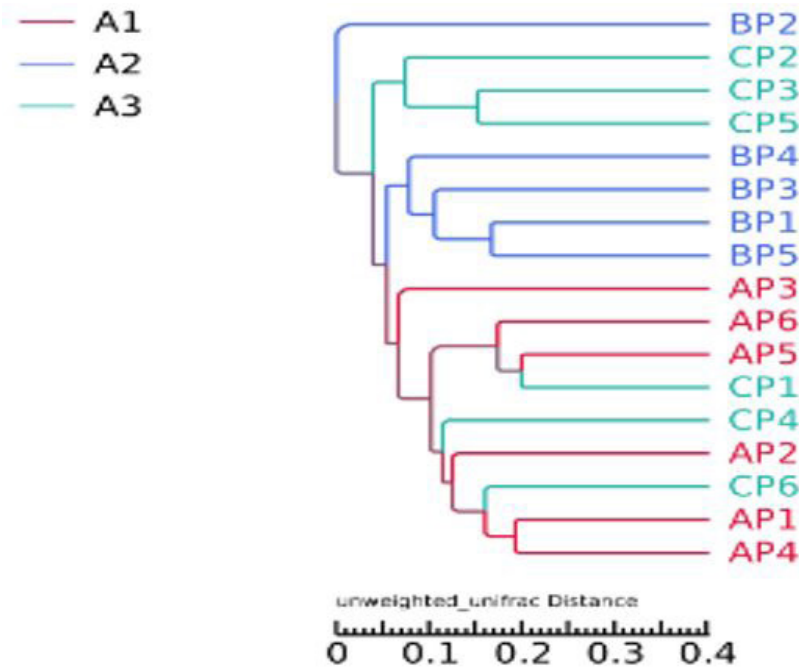

(a)grape

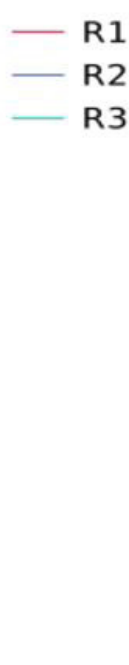

(b)soil

Figure $\mathbf{7 b}$. Cluster analysis diagram of the bacterial communities in the different (a) grape samples and (b) soil samples.

rotation, and different irrigation methods, can change the soil structure and physical and chemical properties, thus altering the composition and distribution of microbial communities (Wawrik et al., 2005). The research results of Baddam et al. showed that bacteria were dominant in vineyard, while fungi were relatively low in abundance (Kamaa et al., 2011). This is consistent with the results of this study. Wei et al. found that
Kaistobacter, Arthrobacter, and Skermanella bacteria existed in a large number of soil samples in the wine-producing areas of the north side of Tianshan Mountain in China. Acidobacteria, Chloroflexi, Gemmatimonadetes, Deinococcus-Thermus, and Candidatus_Saccharibacteria were also found in this study. Renouf et al. showed that the dominant fungi in the soil were Saccharomyces, Sordaria, Tetracladium, and Geomyces, while the 


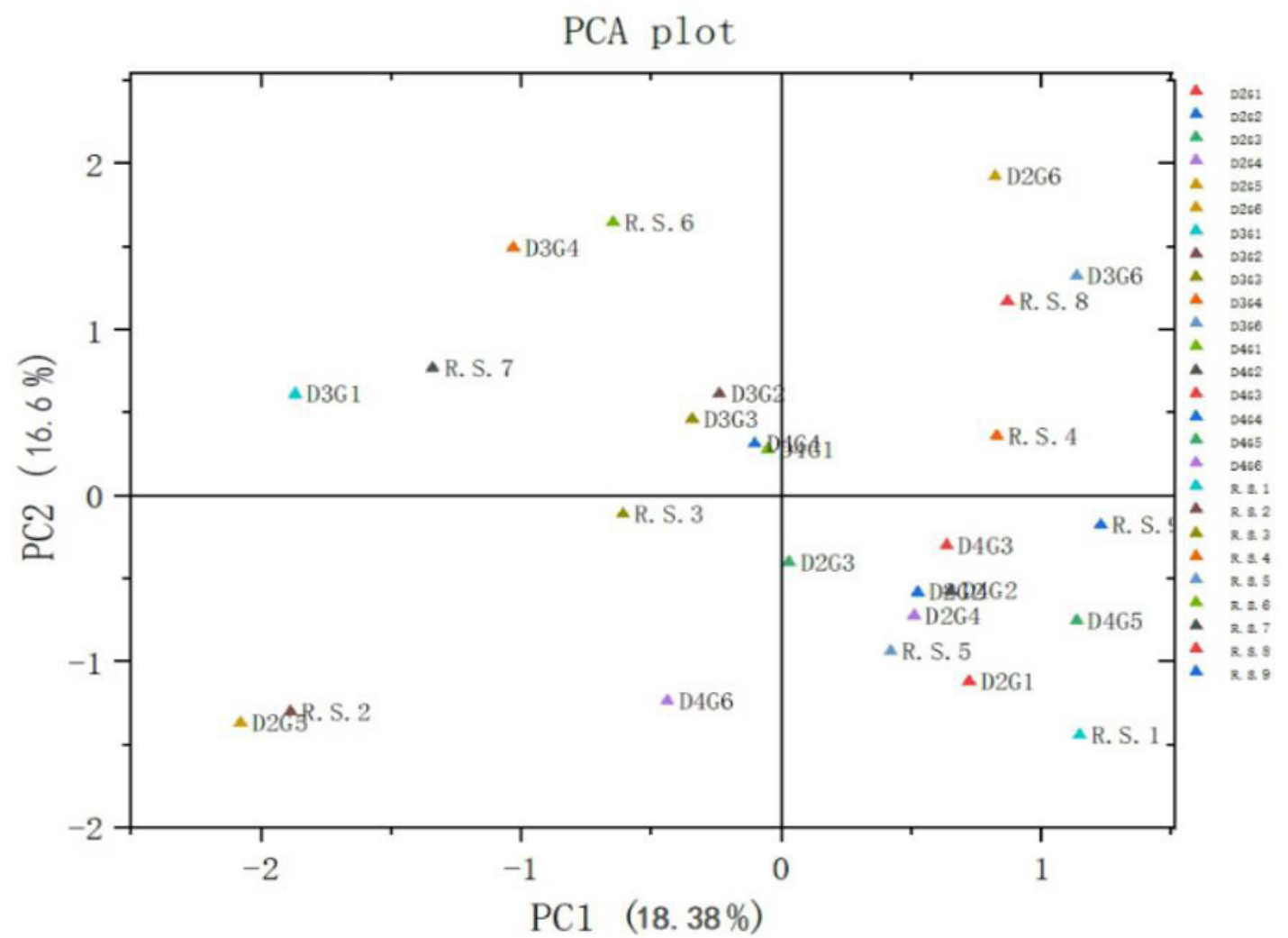

Figure 8a. Principal component analysis plot of the fungal communities in the different samples.

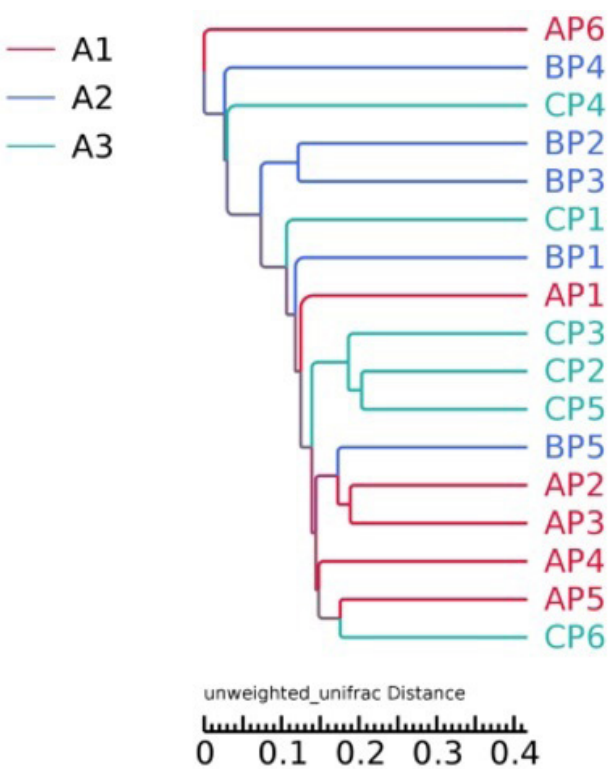

(a)grape
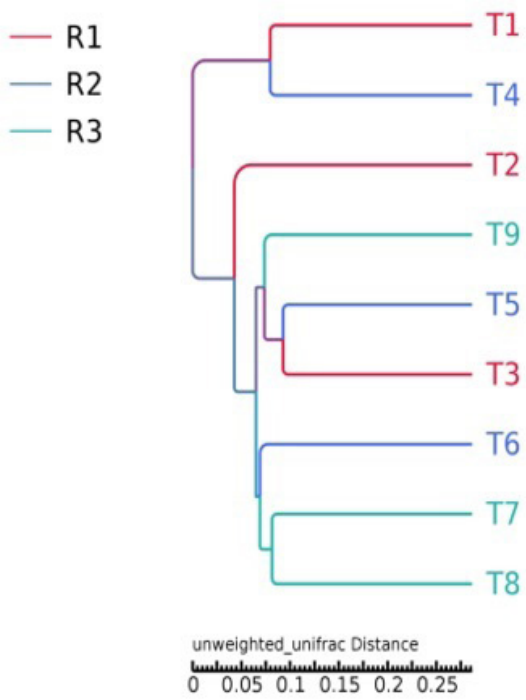

(b)soil

Figure 8b. Cluster analysis diagram of the fungal communities in the different (a) grape samples and (b) soil samples.

dominant fungi in grape and grape leaves were Aureobasidium, Sporospora, Cryptococcus, and Dothideales (Wei et al., 2018). Pinto and Stevanato et al. have shown that Ascomycota and Basidiomycotina are the main fungal microorganisms in soil, grape, and grape leaves (Renouf et al., 2005; Pinto et al., 2014). Wei yujie et al. also found that Saccharomycetaceae, Sordariomycetes, and Tetracladium fungi were abundant in grape samples from wine-producing areas in Xinjiang, China, while Gibberella and Gladaxporism fungi were abundant in the soil samples (Wei et al., 2018). This study detected Ascomycota, Rozellomycota, and Glomeromycota in the soil, as well as Gibberella, Gliomastix, Coprinellu; Zoopagomycota, and Chytridiomycota. The fungi 
Nigrospora, Talaromyces, Filobasidum, Fusarium, and Hyaloscpha were also detected in the grape samples.

The "conventional" yeast extensively used in alcoholic beverage industries is $\mathrm{S}$. cerevisiae due to its tolerance to harsh conditions during alcoholic wine fermentation (Stevanato et al., 2014). Yeast is integral to wine making and directly determines the flavor and sensory quality of wine. At present, there are many varieties of wine-related yeasts, including Saccharomyces, Pichia, Candida, Hanseniaspora, Schizospora, Dekkera, Metschnikowia, Issatchenkia, Saccharomycodes, and Zygosorbimbalces. The most representative is Saccharomyces, which can transform the sugar in grape berries into ethanol and $\mathrm{CO}_{2}$ in alcoholic fermentation (Rêgo et al., 2020), and some non-Saccharomyces cerevisiae can effectively improve the flavor and complexity of wine (Fuelsang \& Edwards, 2007). Among fungal microorganisms,. most harmful filamentous fungi will cause grape quality degradation and reduced production, and wooden barrels or rubber plugs can become easily infected with filamentous fungi during the fermentation. Fusicoccum, which was detected in this study, has the potential to cause a disease that harms vines and infects vine transport tissue, resulting in weakened growth, shrunken leaves, yellowing leaves, or even germination failure and death. This fungus was found in the soil of the GCZ vineyard, and it is recommended that the region strengthen its prevention and treatment of the disease.

LAB are groups of cocci- or bacilli-shaped Gram-positive (Hong \& Park, 2013). The beneficial bacteria mainly included Oenococcus, Latobacillus, and Leuconostoc. Among them, Oenococcusoenosus is the main strain that stimulates MLF. MLF can further reduce the acidity of wine after alcoholic fermentation is complete, and the metabolism of lactic acid bacteria can alter the flavor components in wine so as to improve the sensory quality of wine and increase the mellow feeling of the body (Amelia et al., 2020; Zhang et al., 2020). However, after malic acid is metabolized, lactic acid bacteria will continue to metabolize glycerol and other active ingredients in the body, resulting in rancidity. Therefore, once malolactic fermentation is over, it is necessary to inactivate lactic acid bacteria in time to prevent the body from spoiling. In addition, acetic acid bacteria can convert ethanol into acetic acid, resulting in rancid wine, which is needed to avoid contamination in the wine-making process (Blättel et al., 2009). The composition, quantity and distribution of microorganisms in soil, leaves and fruits of vineyards are affected by many factors, such as climatic conditions, vineyard management mode, phenological characteristics of host plants, soil physical and chemical properties and nutritional status. But only for this research in the vineyards of grape and soil microbial community were studied and liquor-making microbes is the key to affect the wine quality, proven local wine microbial population distribution characteristics in our country has positive significance for the region's wine production, but each region brewing characteristics of microbial resources and its utilization value has yet to be further in-depth study.

\section{Conclusion}

The microbial and fungal diversity of grape berry skins and soils from three vineyards on the foothills of the Helan Mountain region was assessed in the present study. Our findings should inform future wine production in this region by helping establish an excellent wine germplasm repository, assist in the screening of strains, and improve the wine quality of the region.

\section{Acknowledgements}

This work was supported by the National Key Research and Development Project (Item no.2019YFD1002500); Food Science and Engineering Discipline Construction(N0:502218009); the Open Project Program of the Beijing Laboratory of Food Quality and Safety, Beijing Technology and Business University (grant number FQS-201902).

\section{References}

Amelia, R., Philip, K., Pratama, Y. E., \& Purwati, E. (2020). Characterization and probiotic potential of lactic acid bacteria isolated from dadiah sampled in West Sumatra. Food Science and Technology. In press. http://dx.doi.org/10.1590/fst.30020.

Blättel, V., Wirth, K., Claus, H., Schlott, B., Pfeiffer, P., \& König, H. (2009). A lytic enzyme cocktail from Streptomyces sp. B578 for the control of lactic and acetic acid bacteria in wine. Applied Microbiology and Biotechnology, 83(5), 839-848. http://dx.doi.org/10.1007/s00253009-1926-7. PMid:19277643.

Boulton, R. B., Singleton, V. L., Bisson, L. F., \& Kunkee, R. E. (1999). Principles and practices of wine making (pp. 535-542). New York: Springer US. http://dx.doi.org/10.1007/978-1-4757-6255-6.

Cappello, M. S., Zapparoli, G., Logrieco, A., \& Bartowsky, E. J. (2017). Linking wine lactic acid bacteria diversity with wine aroma and flavour. International Journal of Food Microbiology, 243, 16-27. http://dx.doi.org/10.1016/j.ijfoodmicro.2016.11.025. PMid:27940412.

CCB. (2020). flash. Retrieved from http://ccb.jhu.edu/software/FLASH

Cocolin, L., Bisson, L. F., \& Mills, D. A. (2000). Direct profiling of the yeast dynamics in wine fermentations. FEMS Microbiology Letters, 189(1), 81-87. http://dx.doi.org/10.1111/j.1574-6968.2000.tb09210.x. PMid:10913870.

Danielski, G. M., Evangelista, A. G., Luciano, F. B., \& Macedo, R. E. F. (2020). Non-conventional cultures and metabolism-derived compounds for bioprotection of meat and meat products: a review. Critical Reviews in Food Science and Nutrition. In press. http://dx.doi. org/10.1080/10408398.2020.1835818. PMid:33089698.

Drive5. (2020). Muscle. Retrieved from http://www.drive5.com/muscle/

Feng, Y., Motta, A. C., Reeves, D. W., Burmester, C. H., Van Santen, E., \& Osborne, J. A. (2003). Soil microbial communities under conventional-till and no-till continuous cotton systems. Soil Biology \& Biochemistry, 35(12), 1693-1703. http://dx.doi.org/10.1016/j. soilbio.2003.08.016.

Fuelsang, K. C., \& Edwards, C. G. (2007). Wine microbio practical applications and procedures (pp. 44-52). New York: Springer US. http://dx.doi.org/10.1007/978-0-387-33349-6.

Hong, Y. A., \& Park, H. D. (2013). Role of non-saccharomyces yeasts in korean wines produced from campbell early grapes: potential use of hanseniaspora uvarum as a starter culture. Food Microbiology, 34(1), 207-214. http://dx.doi.org/10.1016/j.fm.2012.12.011. PMid:23498200.

Kamaa, M., Mburu, H., Blanchart, E., Chibole, L., Chotte, J.-L., Kibunja, C., \& Lesueur, D. (2011). Effects of organic and inorganic fertilization on soil bacterial and fungal microbial diversity in the kabete long-term trial, kenya. Biology and Fertility of Soils, 47(3), 315-321. http://dx.doi.org/10.1007/s00374-011-0539-3. 
Kioroglou, D., Mas, A., \& Portillo, M. C. (2020). High-throughput sequencing approach to analyze the effect of aging time and barrel usage on the microbial community composition of red wines. Frontiers in Microbiology, 11, 562560. http://dx.doi.org/10.3389/ fmicb.2020.562560. PMid:33013793.

Liu, J., Li, Z., Dong, J., \& Gao, D. (2021). A universal quantification of transgenic soybean event DAS-68416-4 using duplex digital PCR. Journal of the Science of Food and Agriculture, 101(2), 624-630. http://dx.doi.org/10.1002/jsfa.10674. PMid:32687643.

Ma, W., Wu, Y., Wei, Y., Zou, W., Yan, Y., Xue, J., Tian, G., Wang, L., Wang, W., \& Pan, H. (2018). Microbial diversity analysis of vineyards in the xin jiang region using high-throughput sequencing. Journal of the Institute of Brewing, 124(3), 276-283. http://dx.doi. org/10.1002/jib.501.

Martins, G., Miot-Sertier, C., Lauga, B., Claisse, O., Lonvaud-Funel, A., Soulas, G., \& Masneuf-Pomarède, I. (2012). Grape berry bacterial microbiota: Impact of the ripening process and the farming system. International Journal of Food Microbiology, 158(2), 93-100. http:// dx.doi.org/10.1016/j.ijfoodmicro.2012.06.013. PMid:22809638.

Mothur. (2020a). Shannon. Retrieved from https://mothur.org/wiki/ shannon/

Mothur. (2020b). Simpson. Retrieved from https://mothur.org/wiki/ simpson/

Mothur. (2020c). Chao. Retrieved from https://mothur.org/wiki/chao/

Mothur. (2020d). ACE. Retrieved from https://mothur.org/wiki/ace/

Mothur. (2020e). Coverage. Retrieved from https://mothur.org/wiki/ coverage/

Pinto, C., Pinho, D., Sousa, S., Pinheiro, M., Egas, C., \& Gomes, A. C. (2014). Unravelling the diversity of grapevine microbiome. PLoS One, 9(1), e85622. http://dx.doi.org/10.1371/journal.pone.0085622. PMid:24454903.

Prakitchaiwattana, C. J., Fleet, G. H., \& Heard, G. M. (2004). Application and evaluation of denaturing gradient gel electrophoresis to analyse the yeast ecology of wine grapes. FEMS Yeast Research, 4(8), 865-877. http://dx.doi.org/10.1016/j.femsyr.2004.05.004. PMid:15450194.

Qiime. (2020a). split_libraries_fastq.py. Retrieved from http://qiime. org/scripts/split_libraries_fastq.html

Qiime. (2020b).assign_taxonomy.py. Retrieved from http://qiime.org/ scripts/assign_taxonomy.html

Rêgo, E. S. B., Rosa, C. A., Freire, A. L., Machado, A. M. R., Gomes, F. C. O., Costa, A. S. P., Mendonça, M. C., Hernández-Macedo, M. L., \& Padilha, F. F. (2020). Cashew wine and volatile compounds produced during fermentation by non-Saccharomyces and Saccharomyces yeast. LWT, 126, 109291. http://dx.doi.org/10.1016/j.lwt.2020.109291.

Renouf, V., Claisse, O., \& Lonvaud-Funel, A. (2005). Understanding the microbial ecosystem on the grape berry surface through numeration and identification of yeast and bacteria. Australian Journal of Grape and Wine Research, 11(3), 316-327. http://dx.doi. org/10.1111/j.1755-0238.2005.tb00031.x.
Renouf, V., Claisse, O., \& Lonvaud-Funel, A. (2007). Inventory and monitoring of wine microbial consortia. Applied Microbiology and Biotechnology, 75(1), 149-164. http://dx.doi.org/10.1007/s00253006-0798-3. PMid:17235561.

Sarkisian, G. (2019). Research of the properties of grape processing products in relation to its application in spa and wellness industries. Food Science and Technology, 13(1).

Stevanato, P., Bertaggia, M., Stellin, F., Rizzi, V., Piffanelli, P., Angelini, E., Bertazzon, N., Fornasier, F., Squartini, A., Saccomani, M., \& Concheri, G. (2014). Soil biological and biochemical traits linked to nutritional status in grapevine. Journal of Soilence \& Plant Nutrition, 14(2). http://dx.doi.org/10.4067/S0718-95162014005000033.

Verginer, M., Leitner, E., \& Berg, G. (2010). Production of volatile metabolites by grape-associated microorganisms. Journal of Agricultural and Food Chemistry, 58(14), 8344-8350. http://dx.doi. org/10.1021/jf100393w. PMid:20575540.

Wang, C., Tang, J. D., \& Qiu, S. Y. (2020a). Xiao qu profiling of fungal diversity and fermentative yeasts in traditional Chinese. Frontiers in Microbiology, 11, 2103. http://dx.doi.org/10.3389/fmicb.2020.02103. PMid:32983066.

Wang, X., Ren, X., Shao, Q., Peng, X., Zou, W., Sun, Z., Zhang, L., \& Li, H. (2020b). Transformation of microbial negative correlations into positive correlations by Saccharomyces cerevisiae Inoculation during pomegranate wine Fermentation. Applied and Environmental Microbiology, 86(24), e01847-20. http://dx.doi.org/10.1128/ AEM.01847-20. PMid:33036987.

Wawrik, B., Kerkhof, L., Kukor, J., \& Zylstra, G. (2005). Effect of different carbon sources on community composition of bacterial enrichments from soil. Applied and Environmental Microbiology, 71(11), 6776-6783. http://dx.doi.org/10.1128/AEM.71.11.67766783.2005. PMid:16269709.

Wei, Y. J., Wu, Y., Yan, Y. Z., Zou, W., Xue, J., Ma, W. R., Wang, W., Tian, G., \& Wang, L. Y. (2018). High-throughput sequencing of microbial community diversity in soil, grapes, leaves, grape juice and wine of grapevine from china. PLoS One, 13(3), e0193097. http://dx.doi. org/10.1371/journal.pone.0193097. PMid:29565999.

Zhan, B., Fadista, J., Thomsen, B., Hedegaard, J., Panitz, F., \& Bendixen, C. (2011). Global assessment of genomic variation in cattle by genome resequencing and high-throughput genotyping. BMC Genomics, 12(1), 557. http://dx.doi.org/10.1186/1471-2164-12-557. PMid:22082336.

Zhang, J., Wang, L., Shi, L., Chen, X., Liang, M., \& Zhao, L. (2020). Development and application of a real-time loop-mediated isothermal amplification method for quantification of acetobacter aceti in red wine. FEMS Microbiology Letters, 367(19), fnaa152. http://dx.doi. org/10.1093/femsle/fnaa152. PMid:33021644.

Zhao, X., Wang, Y., Cai, W., Yang, M., Zhong, X., Guo, Z., \& Shan, C. (2020). High-throughput sequencing-based analysis of microbial diversity in rice Wine Koji from different areas. Current Microbiology, 77(5), 882889. http://dx.doi.org/10.1007/s00284-020-01877-9. PMid:31950235. 